\title{
INCREMENTALLY ADJUSTABLE ROTOR-BLADE TRACKING TAB USING SMA COMPOSITES
}

\author{
Victor Giurgiutiu*, Craig A. Rogers** \\ University of South Carolina, Department of Mechanical Engineering, Columbia, SC 29208, USA \\ Jasper Zuidervaart ${ }^{\dagger}$ \\ University of Technology, Eindhoven, Netherlands
}

\begin{abstract}
Advanced helicopter rotor systems are designed with non-metallic composite materials for fatigue strength and low radar signature for reduced observability. They incorporate non-metallic composite blades and non-metallic tracking tabs. Adjustment of the existing non-metallic tracking tabs is complex and inefficient since it requires heating and cooling a thermoplastic tab material for each track adjustment. Several track-tab adjustments are required until optimum tracking is achieved.
\end{abstract}

In this paper, a method for achieving direct inflight incremental adjustment of the tracking tab is presented. The method utilizes a special tab material that can be remotely activated by the pilot from the cabin while in forward flight. This material is a composite consisting of shape memory alloy (SMA) wires embedded into a polymeric matrix. The activation of the SMA composite tab is done through electric current controlled from the pilot cabin and sent through conductors along the blade to the tracking tab. The proposed method will achieve in-flight tracking tab adjustment, will minimize the adjustment cycles and will reduce by at least a factor of ten the total tracking time. The implementation of the proposed incrementally adjustable in-flight tracking tab will rapidly minimize the once-per-rev helicopter vibration forward flight. This will lead to reduced crew fatigue, increased component life, and reduced maintenance for the helicopter. Additionally, the proposed method will also be serve as emergency system to reduce unbalanced vibrations due to ballistic damage of the rotor blade .

\footnotetext{
${ }^{*}$ Research Professor, member AIAA, ASME, AHS.

${ }^{* *}$ Professor of Mechanical Engineering and Dean of the College of Engineering, member AIAA, ASME.

${ }^{\dagger}$ Summer Intern, Center for Intelligent Material Systems and Structures, Virginia Tech, Blacksburg, VA 24061-0261.
}

Copyright (C) 1997 by V. Giurgiutiu and C. A. Rogers. Published by the American Institute of Aeronautics and Astronautics, Inc. with permission

\section{INTRODUCTION}

\subsection{Background}

Helicopter rotor blades production process is subject to manufacturing tolerances. Blade's mass, center of gravity position, and aerodynamic contours may vary from blade to blade according to the overall accepted tolerances. With reinforced plastics (composite) blades these variations are quite significant due to the inherent nature of this manufacturing process.

Once the blades are mounted in the helicopter rotor assembly (typically 4 blades per rotor) the inherent differences between blades may lead to significant imbalance problems leading to unacceptable behavior in operation, especially regarding $1 / \mathrm{rev}$ vibrations (Figure 1). These vibrations are detrimental to the ride comfort, to the safe and accurate handling of the cockpit instrumentation, to the operation of the weapons aiming systems, and generally produce dynamic loads detrimental to the fatigue life of the aircraft. Furthermore, these vibrations increase several times with the helicopter forward speed, due to phenomena related to flow asymmetry and harmonic motion of the blade.

Tracking tabs are used on most helicopter rotor blades to reduce vibration in forward flight. Conventional rotor blade tracking tabs are thin aluminum plates located on the blade trailing edge. These tabs are adjusted with a calibrated bending tool to alter the track of individual blades, thereby compensating for small manufacturing differences between the individual blades of the rotor. When the track of all blades are nearly identical, the once-per-rev vibration of the rotor is minimized, leading to reduced crew fatigue, increased component life, and reduced maintenance for the helicopter. However, a number of tracking tab adjustment cycles (take off, forward flight evaluation, landing, and readjustment) are required before the optimal track is achieved. Besides, the conventional tracking tabs are passive and hence 
rely on correcting the problem on the ground. They cannot properly address the forward flight vibrations, which are actually the most detrimental in helicopter operation.

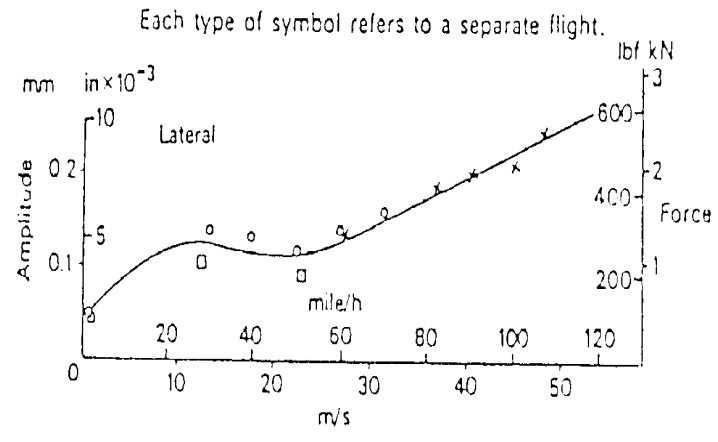

Figure 1 Spectrum of vibration amplitudes and effect of airspeed for a three-blade helicopter rotor (after Bramwell ${ }^{1}$ ).

A better way to address the problem would be to have an active device that permits in flight trim of the blade, thus addressing the vibrations that dependent on forward flight conditions. Additionally, such a device will replace some of the lengthy and man-power intensive steps in the existing conventional techniques. Thus lower cost, and higher productivity will be achieved. Finally, the device will also be able to deal with ballistically produced imbalance, a subject so far unexplored by other investigators. Opportunities for such novel devices arise with the advent of active materials technology that offer numerous applications in the aerospace industry ${ }^{2,3}$.

In this paper, a method for achieving direct inflight incremental adjustment of the tracking tab is presented. The method utilizes a special tab material that can be remotely activated by the pilot from the cabin while in forward flight. The special tab material used in this proposal is a composite consisting of shape memory alloy (SMA) wires embedded into a polymeric matrix. The activation of the SMA composite tab is done through electric current controlled from the pilot cabin and sent through conductors along the blade to the tracking tab. The proposed method will achieve in-flight tracking tab adjustment, will minimize the blade tracking cycles and will reduce by at least a factor of ten the total tracking time.
1.2. Existing Conventional Techniques for Reducing Blade Imbalance Vibrations

One conventional approach for reducing blade imbalance vibrations has been to tighten tolerances imposed on the manufacturing process (both on mass and CG location, and on the aerodynamic contours of the blade section). This has been shown to alleviate somehow the phenomenon, but has lead to substantial increase in rejection rates, and hence in manufacturing costs. Additionally, increased inspection requirements have required more personnel and more sophisticated equipment, thus further escalating the cost.

Another conventional approach currently used in the industry has been to correct the phenomenon through mass balance and aerodynamic tabs. A two stage process is currently used:

First the blades are statically balanced at the end of the production line. Fixing bolts are commonly provided under a cowl at the tip of the blade; with the cowl remover, various balancing weights can be added to compensate for the outof-tolerance values of mass and CG position. Highly sensitive balancing machines are used in this operation, and the process is highly mechanized and efficient. To allow for "negative" mass balance (i.e. the blade mass exceeds the mean statistical value) some permanent mass balance weights are designed into all blades, thus allowing for addition or subtraction of weight, as the need may arise.

Secondly the blades are dynamically balanced in the whirl tower. The blades are mounted in a real rotor hub assembly on top of a fixture (whirl tower) allowing for rotation at variable speeds up to, and a few percentages above the operational RPM of the helicopter. Next the rotor is spinned up at increasing speeds, and attention is paid to the blade tip movement around the circumference (blade tracking). Sophisticated, highly computerized tracking devises are used in this operation. Out of track behavior, both in bending (flap or lead-lag) and in torsion is identified and traced back to the changes required in the value and/or position of the balancing weights at the tip of the blades. Fine adjustments are made to correct unwanted behavior and a higher spinning speed is next attempted. This iterative process is repeated until operational speed (and also a few percentages above it, for margin of safety reasons) is reached. 
( For improved dynamic behavior, some helicopter manufacturers even require that the blades are balanced in quadruple sets, that subsequently stay together for the rest of their operating life. If one of the blades is accidentally damaged during service, then the complete set of blades mounted on that particular aircraft will have to be changed.)

Aerodynamic difference between blades are reconciled in a similar manner. A fix trailing edge metal tab is provided towards the tip of each blade, and in an iterative aerodynamic balancing process these tabs are modified (usually through permanent plastic deformation) until all 4 blades track in the same circumferential trajectory.

\subsection{Disadvantages of Existing Blade Trimming Methods}

The existing rotor blade trimming methods have a series of disadvantages:

(i) High manufacturing costs: It is obvious that conventional dynamic and aerodynamic balancing processes are lengthy and labor intensive, contributing a significant amount to the cost of the blade and hence of the complete aircraft.

(ii) Need for periodic re-balancing: Another significant aspect is that during service small changes may appear in the dynamic behavior of individual blades, even more so with composite construction. Blade sets that were initially "perfectly" balanced tend to develop in service imbalance that leads to unwanted and detrimental vibrations, and may require replacement while still having unexpired fatigue lives.

(iii) Ballistic damage aspects: Battlefield small arms fire may cause blade damages which do not render the blade non-operational from the strength point of view. However, the accompanying imbalance, producing severe vibrations and even air resonance phenomena, can be a very serious problem that significantly reduces the battle effectiveness of the aircraft, and under extreme conditions can lead to the loss of the aircraft and/or crew. So far the pilot is not able to do anything to counteract such situations and usually has to abandon battle and get to the ground base.

\subsection{Proposed Improvement of Rotor Blade Tracking}

We propose to explore and develop a practical method for remotely trimming the rotor blade and thus counteract the aerodynamic imbalances discussed above. Such a method should have the following operational goals:

(i) permit the modification of aerodynamic trim of individual blades through remote action, and during blade rotation;

(ii) allow the pilot or ground crew to do periodic mass balance adjustments to correct for balance drift appearing under normal operating conditions;

(iii) allow the pilot to undertake in-flight corrective actions for counteracting the imbalance effects of small arms fire damage on the blade;

(iv) operate automatically in an active control mode for alleviating general $1 / \mathrm{rev}$ vibrations appearing during the flight regime.

Conventional actuation methods, like miniaturized hydraulic cylinders or electric motors, are unlikely to give a satisfactory solution to this problem due to high cost, complexity of power supply, and difficulty to operate in the high-g environment present at the blade tip. A solid state device would be preferred for this application for obvious reasons. Such a solid state solution would be the active composite material obtained by embedding shape memory alloy (SMA) wires into polymeric composites. Giurgiutiu et al. ${ }^{4}$ has recently reported preliminary experimental results with laboratory scale SMA composites that give promising perspectives for implementation in a helicopter blade active tracking tab system. This solid state actuating material does not have many of the disadvantages of other solutions based on discrete actuators and mechanical linkages.

\section{SHAPE MEMORY EFFECT AND SHAPE MEMORY ALLOYS (SMA) MATERIALS}

Numerous authors have proposed actuation solutions based on the use of shape memory alloy (SMA). Applications range from vibrations control of plates ${ }^{5,6}$, through structural modifications of composite response ${ }^{7,8}$, to active shape control ${ }^{9,10}$. A brief description of the shape memory effect and it potential for actuation applications is given next.

\subsection{Induced-Strain Actuation with Shape Memory Alloys}

Shape memory alloys (SMA) materials are thermally active materials that undergo phase transformation when the temperature passed 
predetermined threshold values. When phase transformation takes place, the SMA material modifies its shape, i.e., it has memory. Details of this process are given next.

\subsubsection{Temperature-triggered strain recovery in shape memory alloys}

A number of special alloys display the property of strain recovery through temperature-triggered phase-transformation ${ }^{13-16}$. At lower temperatures, these alloys are in martensitic state, while, at higher temperature, they are in austenitic phase. Upon heating, the change from martensitic phase to austenitic phase starts at temperature $A_{s}$ and finishes at temperature $A_{\mathrm{f}}$. Upon cooling, the change from austenitic phase to martensitic phase starts at temperature $M_{s}$ and finishes at temperature $M_{\mathrm{f}}$. Note that $A_{s}<A_{\mathrm{f}}$, while $M_{s}>M_{\mathrm{f}}$. The values of the phase-transition temperatures can be varies with alloy composition. Shape memory alloys with operating range between $50^{\circ} \mathrm{C}$ and $100^{\circ} \mathrm{C}$ are currently available (e.g. NiTi Material \#1 in Table 1).

When these materials undergo phase transformation, they may display the shape memory alloy (SMA) effect. Assume that we work with an SMA material which is the martensitic phase at room temperature. While the SMA is in the martensitic phase, we deform it to a large permanent strain (typically, 5-10\%). This permanent deformation of the material in the martensitic phase is basically achieved through a permanent deformation of the crystalline lattice (twinning). Next, assume that the SMA material is heated. At temperature $A_{s}$, the phase transformation is triggered and the internal metallographical structure starts changing from the martensitic into the austenitic.

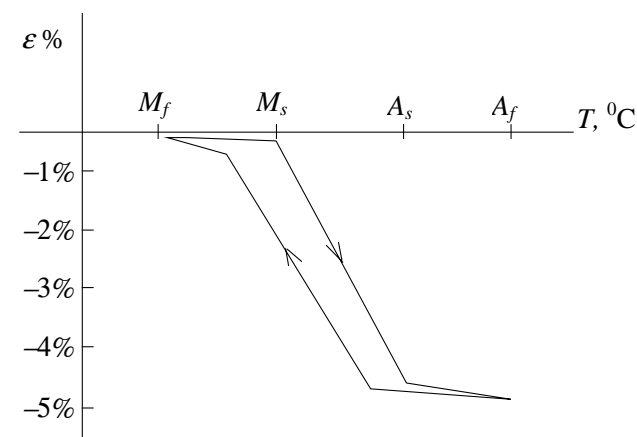

Figure 2 Schematic representation of strain recovery through the SMA effect in a two-way shape memory alloy (after Lagoudas ${ }^{13}$ ).

As the phase transformation takes place, the crystalline lattice undergoes "detwinning", i.e., it reverts back to the original state. Thus, the material recovers the initial shape that it had before the permanent strain deformation was applied. This strain recovery process is named "shape memory". If the temperature is now decreased, the material returns again to the martensitic phase, and the lattice becomes again twinned. However, hysteresis is involved, as shown schematically in Figure 3.

Please note that the SMA material illustrated in Figure 4 displays the two-way shape memory effect. These alloys assume one shape when in the martensitic phase, at lower temperatures (strain state $M$ ), and another shape when in the austenitic phase, at higher temperatures (strain state $A$ ). Upon heating and cooling the material can be cycled between the two shapes (strain states). Other shape memory materials only display the one-way shape memory effect. They do not return to the deformed shape when, upon cooling, the reverse phase transformation from austenitic to martensitic state takes place.

Table 1: Properties of some shape memory alloys (after Barrett ${ }^{16}$ )

\begin{tabular}{cccc}
\hline \hline Property & $\begin{array}{c}\text { NiTi } \\
\text { Material \#1 }\end{array}$ & $\begin{array}{c}\text { NiTi } \\
\text { Material \#2 }\end{array}$ & $\begin{array}{c}\text { Copper SMA } \\
\text { Material \#3 }\end{array}$ \\
\hline Martensitic finish temperature, $M_{f}\left({ }^{0} \mathrm{C}\right)$ & 23 & 5 & -34 \\
Martensitic start temperature, $M_{s}\left({ }^{0} \mathrm{C}\right)$ & 50 & 23 & -27 \\
Austenitic start temperature, $A_{s}\left({ }^{0} \mathrm{C}\right)$ & 62 & 29 & -25 \\
Austenitic finish temperature, $A_{f}\left({ }^{0} \mathrm{C}\right)$ & 100 & 51 & -14 \\
Martensitic Young's modulus, $E_{M}(\mathrm{GPa})$ & 13 & 13 & 7 \\
Austenitic Young's modulus, $E_{M}(\mathrm{GPa})$ & 30 & 30 & 7 \\
Martensitic CTE, $\alpha_{A}\left(10^{-6} \rho^{0} \mathrm{C}\right)$ & 11 & 11 & 14.3 \\
Austenitic CTE, $\alpha_{A}\left(10^{-6} /{ }^{0} \mathrm{C}\right)$ & 11 & 11 & 14.3 \\
\hline \hline
\end{tabular}


To repeat the shape memory affect, such oneway SMA materials must be again permanently strained while in the martensitic phase. To achieve a two-way motion with a one-way SMA materials, either a bias spring arrangement, or a counteracting SMA construction with alternative activation, can be employed.

\subsubsection{Improved SMA effect through training}

Training is a process through which the SMA effect is repeatedly applied to the material. Through training, the SMA properties of the material stabilize and become more predictable and repetitive. Lagoudas ${ }^{13}$ describes the twoway training process consisting of the following steps:

1. Strain the wires to $10-11 \%$

2. Release the wire from the straining device

3. Heat the wire until fully transform into austenite

4. Repeat steps 1 - 3 at least twenty times, always calculating strain from the wire length during the previous cycle.

Training can be applied to the material prior to use, or in situ, after fabrication of the SMAcontaining part. In situ training is used when the manufacturing of the part incorporating SMA materials requires temperatures above the phase transition temperature of the SMA material.

\subsubsection{SMA energy available for external work}

The modeling of the internal mechanism of the SMA effect has been the subject of a large number of scientific papers available in the specialized literature. Some of the mathematical developments of these papers are extensive, since they aim at capturing several aspects such as the partial transition from one phase into the other, the plasticity of the material, strain-rate effects and thermal-expansion strains. However, for the present proposal it is sufficient to present a very simplified concept, viz. the SMA induced-strain, $\varepsilon_{\mathrm{SMA}}$, and the accompanying stored energy that can be made available for performing mechanical work ${ }^{20}$.

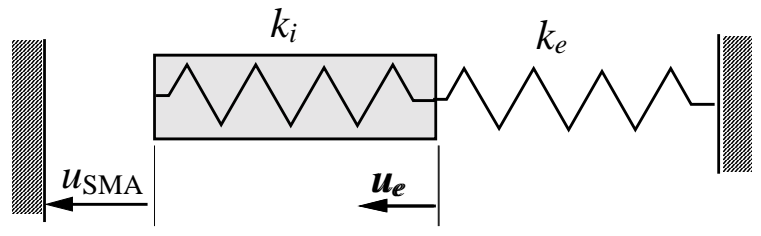

Figure 5 Schematic representation of SMA structural actuation

Assume an SMA actuator consisting of an SMA wire of diameter $D$, cross-sectional area $A=\pi D^{2} / 4$, and length $L$. Hence, its internal stiffness, $k_{i}$, is given by: $k_{i}=\frac{E L}{A}$. On the other hand, the effective stiffness of the actuated structure, at the point of action of the SMA actuator, is $k_{e}$. As the SMA effect is applied, the SMA actuator undergoes a shrinking displacement, $u_{\mathrm{SMA}}$. This displacement is transmitted to the actuated structure, less an amount lost inside the SMA actuator due to its internal elasticity represented by $k_{i}$. The effective structural displacement is,

$$
u_{e}=\frac{1}{1+k_{e} / k_{i}} u_{\text {SMA }}
$$

This equation shows that only a limited amount of SMA displacement reaches the actuated structure. Similarly, one can trace the energy that gets transmitted to the actuated structure and the energy that remains inside the actuator due to its internal elasticity. As shown by Giurgiutiu, Chaudhry and Rogers ${ }^{20}$ the maximum energy that can be made available to the structure is obtained when the stiffness is matched $\left(k_{e}=k_{i}\right)$, and it value is

$$
\left(E_{e}\right)_{\max }=\frac{1}{4} k_{i}^{2} u_{\mathrm{SMA}}
$$

\subsubsection{SMA structural actuation}

The principles of structural actuation based on the SMA effect consist in utilizing the SMA stored energy for deforming the structure upon the application of thermal triggering. The most effective way to implement this is by using the axial SMA effect obtained in SMA wires that can be embedded into a built-up structure, or applied to an existing structure. At roomtemperature, the SMA wires are in the martensitic state. By training the martensiticstate wires at around 10\% tensile strains, a certain amount of energy gets stored into the twinned martensitic lattice. When the SMA wires 
are heated, as for example by passing an electric current through them, phase transformation into the austenitic phase produces strain recovery, basically shrinkage of the SMA wires. As the SMA wires shrink, they deform the structure to the desired shape, using the mechanics of eccentric tension-bending. Chaudhry and Rogers ${ }^{9}$ presented the results of some very illustrative experiments with lateral bending of beams and struts using eccentrically attached SMA wires.

\section{Preliminary Tests of a SMA COMPOSITE PLATE}

Laboratory tests on a proof-of-concept demonstrator SMA-composite plate, performed by Giurgiutiu et al. ${ }^{4}$ produced preliminary results:

(a)

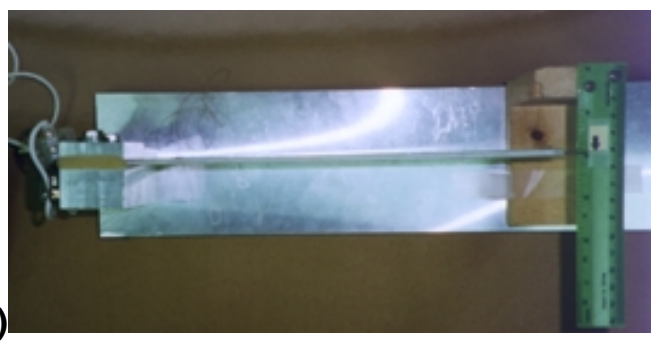

(b)

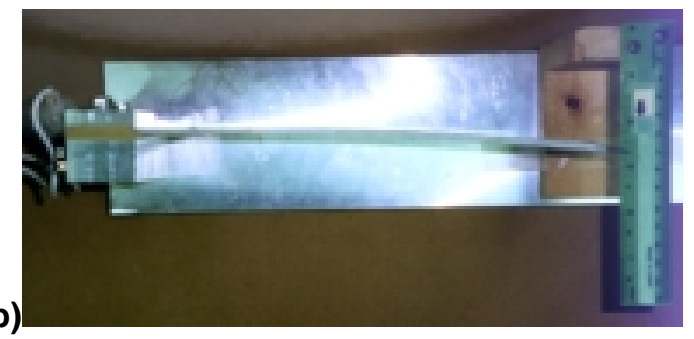

Figure 6 Deformation of SMA composite plate during laboratory tests: (a) before activation; (b) after activation of the SMA effect.

The SMA-composite plate was capable of largeamplitude tip deflections $(\sim 35 \mathrm{~mm})$ when thermally activated above the $A_{f}$ temperature by passing an electric current through the SMA wires (Figure 4). Control of the tip deflection via modification in the electric input (voltage or current) was not found to be a feasible practical solution. The heat flux at various ambient conditions was found difficult to control, and hence the precise control of the SMA temperature in the $A_{s}-A_{f}$ range was not possible. Hence, it was decided that a feasible implementation of the concept will rely on a "ON-OFF" concept of SMA activation.

\section{Proposed Concept for InCRementally ADJUSTABLE HELICOPTER ROTOR BLADES TRACKING TAB USING SMA COMPOSITES}

Figure 5 presents the proposed concept for incrementally adjustable helicopter rotor blade tracking tab using an SMA composite design. The tab is fixed to the trailing edge of the blade section over a span of several inches toward the blade tip. The location and dimensions of the tab will be similar to those of existing tabs.

The principle of operation of the incrementally adjustable helicopter rotor blade tracking tab is based on the strain recovery effect in the SMA wires. Each wire is "charged" with a certain amount of strain energy that is released upon thermal activation of the phase transformation process. Upon thermal activation, the SMA wires undergo phase transition and shorten their length in proportion to the induced strain, $\varepsilon_{S M A}$. Subsequently, the tab will undergo bending deformation. Figure 1 presents a sketch of the tab deformation when the SMA wires have been activated. In this sketch, the SMA wires on the top-side were assumed activated, and hence the tab bends upwards. The bending of the tab is the result of asymmetric shrinking by the induced strain, $\varepsilon_{S M A}$. Resolved about the neutral axis, the induced strain, $\varepsilon_{S M A}$, generates an inplane action and a bending action. The in-plane action is being resisted by the base plate, while the bending action produces upwards deformation of the tab. It should be noted that, during this process, the SMA wires on the bottom surface are not activated. The bottomsurface SMA wires, which play a passive role during the upwards deformation of the tab, store part of the induced-strain energy released by the activation of the top-side wires. To achieve downwards tab deformation, the bottom-side SMA wires are activated. A simplified version of the SMA composite tab, in which SMA wires are only placed on only one side of the tab, can also be constructed. Such a simplified tab version will have a higher deformation effectiveness when activated, since there will be no passive SMA wires on the opposite side to take part of the induced-strain energy. However, the simplified tab version will be able to deform into only one direction. Control of SMA actuation can be achieved through several mean. One way that was previously proposed of controlling the amplitude of SMA actuation is to implement only a limited amount of strain recovery by inducing only a partial martensitic-to-austenitic phase transformation. 
To achieve this, very precise temperature control is necessary, which is not always practically possible. A second proposed way of controlling the amplitude of SMA actuation is to vary the amount of strain initially stored in the SMA wires during training. However, this method does not lend itself easily to implementation. A third possible way of controlling the amplitude of SMA actuation is to have separate sets of wires that can be independently activated. This independent activation could be achieved through separate electric circuits. Thus, a stepwise activation process is envisioned. The only draw back of this control method could be the thermal crosstalk between the activated and the not-activated SMA wires. A fourth way of producing controlled incremental tab motion is to split the tab into sections that are sequentially activated. Then, each section will produce a fraction of the total aerodynamic effect. These two last options will be explored next.

\subsection{Geometric Deflection Control Through Selective Activation of Embedded SMA Wires}

One way to achieve incremental tab motion is through incremental activation of selected SMA wires inside the SMA composite. Thus, at activation level \#1, only one out of four wires are activated; at level \#2, two out of four; at level \#3, three out of four: and at level \#4 (full level) all wires are activated. The selection of activation direction, i.e., either "up" or "down", is achieved by activating either the wires on the top surface, or the wires on the bottom surface.

\subsection{Aerodynamic Deflection Control Through Selective Activation of Tab Split Sections}

For practical implementation, it seems more feasible and reliable to consider a simplified SMA-composite plate concept that will only produce "ON-OFF" displacement. In order to achieve a tracking tab with incremental operation, we developed the concept of using a battery of SMA-composite plate elements and then activating them sequentially. This concept is illustrated in Figure 7. Note that two options are presented: one option consists of an 8element tracking tab, the other option consists of a 16-element tracking tab.
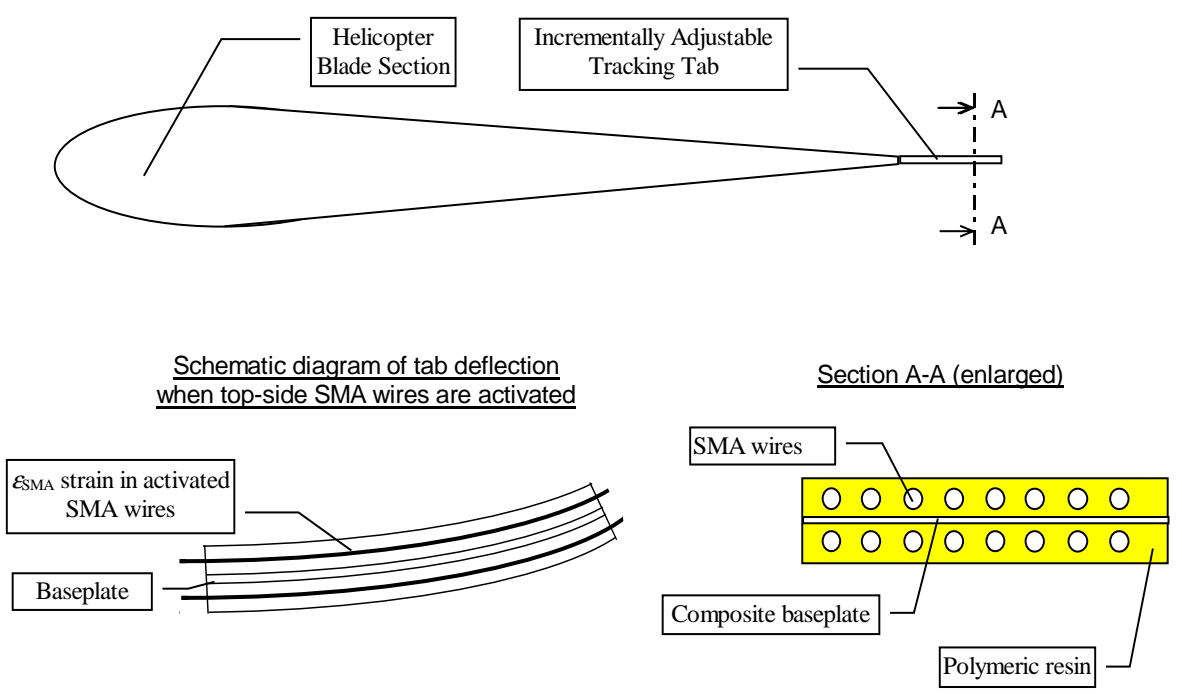

Figure 8 Proposed concept of incrementally adjustable rotor tracking tab using an active-shape composite material consisting of SMA wires embedded in polymeric resin on both sides of a central composite baseplate.

The 8-element tracking tab represents the basic configuration, and it is similar to the conventional tracking tab currently installed on the MD-900 helicopter blade ${ }^{21}$. This tab has a total deflection of $\pm 7.5^{\circ}$ that can be activated incrementally in 8 steps by sequentially 
activating the 1 "-long elements. Since the area of each element is $1 / 8$ of the total tab area, the effective aerodynamic effect of deflecting one element is $1 / 8$ of the aerodynamic effect of deflecting the complete tab. Hence, the deflection of each of the 8 elements has the same effect as the deflection of the complete tab by $1 / 8$ of its maximum angle. A simple calculation shows that this concept gives an incremental step of approximately 1 .

(a)

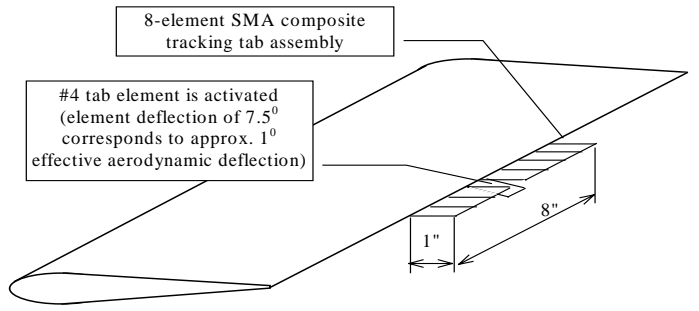

Plan view of the 8-element adjustable tracking tab

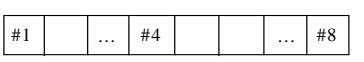

(b)

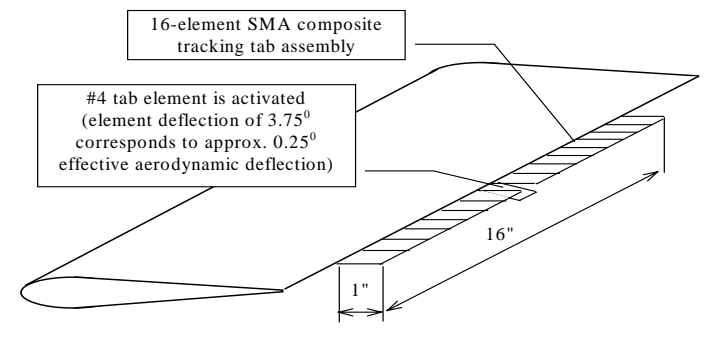

Plan view of the 16-element adjustable tracking tab

\begin{tabular}{|l|l|l|l|l|l|l|l|l|l|l|l|l|l|l|l|}
\hline$\# 1$ & & & $\ldots$ & & & & $\# 8$ & & & & $\ldots$ & & & & $\# 16$ \\
\hline
\end{tabular}

Figure 9 Two concepts for multi-element incrementally adjustable tracking tab: (a) 8-element tab with $1^{0}$ equivalent incremental deflection of in the range $\pm 7.5^{0}$; (b) 16 -element tab with $1 / 2^{0}$ incremental deflection in the range $\pm 3.75^{\circ}$. Both options can give the same aerodynamic, but option (b) offers finer tuning.

For a finer tuning capability, the concept of the 16-element tracking tab can be used. Since this tracking tab is twice as long as the baseline configuration, it only needs to deflect only about half as much in order to achieve the same aerodynamic effect. Hence, its maximum deflection would be around $3.75^{\circ}$. Considering that this 16 "-long tracking tab is made of the same 1"-long elements as the 8"-long tab, the activation of one element will result in an increment of approximately $14^{0}$, It can be seen that the 16"-long tracking tab gives a finer tuning capability than 8"-long tab. Additionally, it has the benefit of requiring a lower displacement from the SMA composite element.

\section{Practical IMPLEMENTATION OF THE SMA COMPOSITE TAB CONCEPT}

The practical implementation of the SMA composite tab concepts requires technological solution to several problems. Two of these, the tab construction method and the tab operation, are detailed next.

\subsection{Proposed Construction of the SMA Composite Tab for Rotor Blade Tracking}

The structural construction of the incrementally adjustable helicopter rotor blade tracking tab consists of SMA wires embedded in a polymeric resin. Figure 5 shows an enlarged view of the tab cross-section. An equal number of SMA wires are placed, equally spaced, on both sides of a thin composite base plate. Before placement, the SMA wires are "trained" in bulk to approximately $5 \%$ "locked-in" strain. The locked-in strain existing in the wires will produce, upon selective thermal activation, the incremental bending deformation of the tab. The base plate and the SMA wires are embedded in a polymeric resin. The application of the embedding polymeric resin can be done through a variety of methods, depending of the chemical specification of the resin system. Liquid casting, solution application in layers, or powder technology can be used, as appropriate. In the initial phase, a room-temperature application will be used, such that the SMA wires are kept below the phase-transition temperature.

For the production phase of the SMA composite tab for helicopter rotor blade tracking, further refinements in the selection of the polymeric and SMA material systems can be implemented. The chemical system of the polymeric material can be selected to be the same, or at least compatible, with the resin system used in the construction of the composite helicopter blade on which the SMA tab will be eventually installed. Thus, the production SMA tab will be incorporated directly into the blade structure. The glass-transition temperature of the polymeric resin and the phase-transition temperature of the SMA wires will be selected in such a way that the thermoplastic resin will not soften excessively while the SMA wires are 
being thermally activated. Additionally, the SMA phase-transition temperature must be substantially above the environmental temperature of helicopter operation, such that inadvertent activation of the SMA effect is prevented.

\subsection{Operation of the SMA Composite Tab for Rotor Blade Tracking}

The SMA composite tab is activated by electric current passed through the selected SMA wires. The electric current generates a temperature rise in the SMA wires through resistive heating. In the initial development of the SMA composite tab, the temperature inside the SMA composite tab is prevented from rising above the glasstransition temperature of the thermoplastic resin through a temperature controller and a temperature sensor (thermocouple probe). This temperature control sub-system may be eliminated later on in the system development cycle through calibration of the current requirement as function of the environmental temperature outside the helicopter.

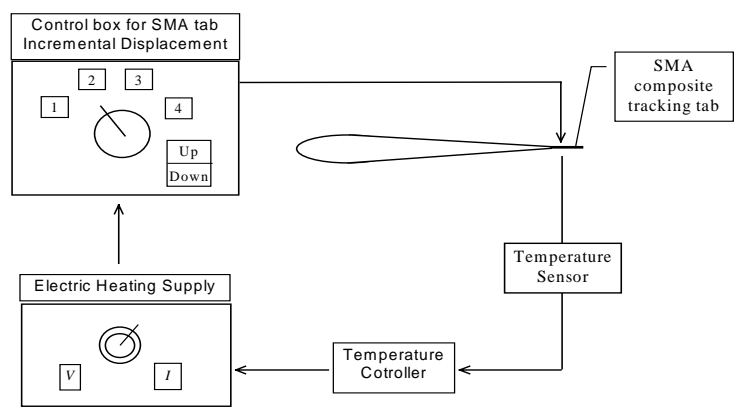

Figure 10 Conceptual system diagram for incrementally ad

The operation of the tab is done through a control box that has several incremental positions. Figure 7 shows an example with four increments, from \#1 to \#4. As the selector is moved from \#1 towards \#4, the effective aerodynamic deflection of the tab increases. A switch is also provided for changing the direction of motion from "up" to "down". The control box for the incrementally adjustable helicopter rotor blade tracking tab is placed in the pilot cabin, hence the system can be operated with the helicopter on the ground or even in flight. For tracking production blades, operation of the incrementally adjustable tracking tab is also possible, if desired, in the whirl tower.

\section{HELICOPTER INSTALLATION OF THE SMA- COMPOSITE ROTOR BLADE TRACKING TAB}

A schematic of the helicopter installation concept for the incrementally adjustable SMA composite tab system is given in Figure 8 . The SMA composite tabs are placed on the rotor blades (only one blade is shown in Figure 8), while the control electronics are place in the helicopter fuselage. The SMA tabs are activated electrically through cables passed along the blade. (Production versions of the incrementally adjustable SMA composite tab system may have the cables passed through blade spar during the blade-fabrication process.) In the experimental stage of product development, standard slip rings may be used to transmit the activation signals from the fuselage control box to the blade SMA tabs. In further developments of the concept, it is envisioned that a more compact solution can be developed. For example, electric power generation in rotating frame, i.e., on the rotor blade hub, can be considered. In this case, the high current signals needed for tab activation will be produced in rotating frame, while the control signals can be sent through teletransmission.

\section{FLIGHT TRIMMING OF HELICOPTER ROTOR BLADES USING THE SMA-COMPOSITE TAB}

Operation of the SMA composite tab for tristamiegrawh grtánpaired blades is performed is performed step wise. The pilot, activates the tab incrementally, and monitors the blade and rotor vibrations at each tab incremental position, until the best tracking condition is achieved. At the end of the tracking cycle, the best-compromise deformed position of the tab for a particular flight condition has been achieved. Then a next flight regime must be considered. For a general procedure, three phases have to be considered: ground trim, hover flight trim, and forward flight trim. 


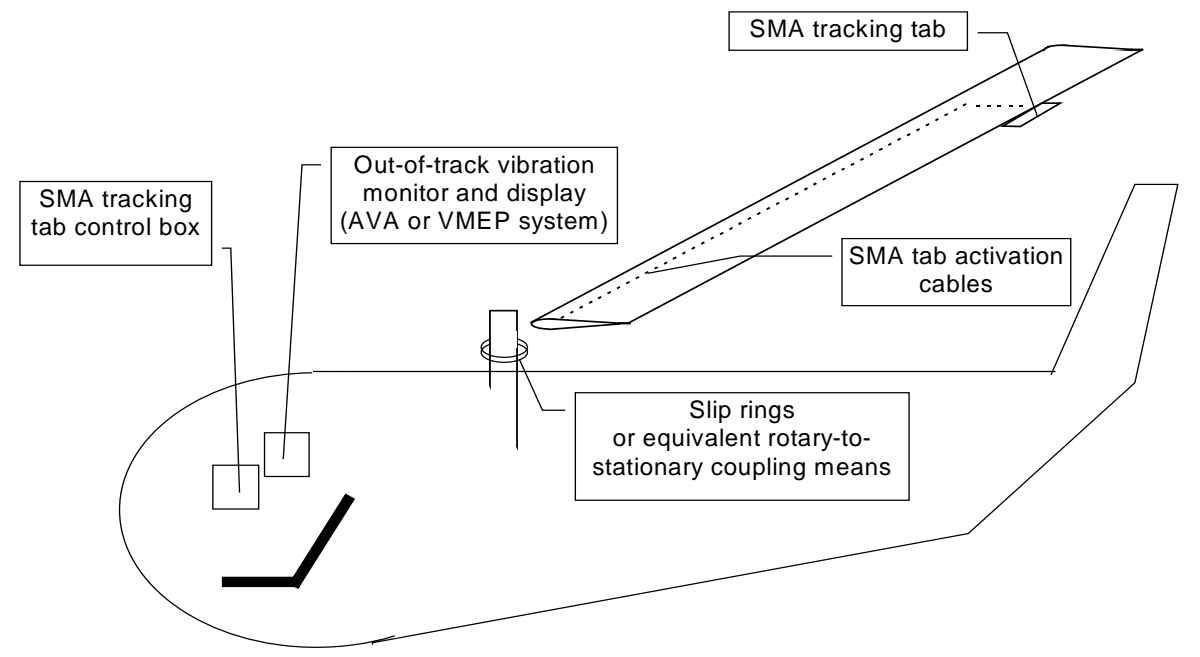

Figure 11 Proposed concept for helicopter installation of incrementally adjustable rotor tracking tab utilizing SMA active-composite materials.

\subsection{Ground Trim}

1. Start rotor and bring to speed

2. Measure vibration level and apply criteria to decide if trim is necessary.

3. Activate SMA tab and slowly sweep through the possible settings on the Tab Control Box. Identify the setting with least vibration level. (For future development, microprocessor control of this process will ensure that the setting that gives least vibration is identified).

4. Desactivate SMA tab and stop the rotor.

\subsection{Hover Flight Trim}

1. Bring helicopter to hover flight.

2. Measure vibration level and apply criteria to decide if trim is necessary.

3. Activate SMA tab and slowly sweep through the possible settings on the Tab Control Box, while keeping the hover regime constant. Identify the setting with least vibration level.

4. Desactivate SMA tab

5. Bring the helicopter into landing.

\subsection{Forward Flight Trim}

1. Bring helicopter to forward flight regime.

2. Measure vibration level and apply criteria to decide if trim is necessary.

3. Activate SMA tab and slowly sweep through the possible settings on the Tab Control Box, while keeping the forward flight regime constant. Identify the setting with least vibration level.
4. Desactivate SMA tab.

5. Repeat \#2 through \#4 for the other forward flight regimes of the helicopter.

\subsection{Permanent Trim Adjustment}

After all the trim conditions have been investigated, the SMA tab can be permanently set into the position that gives an optimal low level of vibrations for all flight regimes. This permanent setting can be achieved by activating the SMA setting that was found to be optimal, and then bringing the thermoplastic material of the tab over the glass transition temperature using a heat gun to "locked in" the optimal shape of the tab. Subsequently, the tab becomes inactive, until a change of blade properties, through service fatigue, battle field action, or otherwise, requires a new tracking cycle.

\section{CONCLUSIONS}

A method for achieving direct in-flight incremental adjustment of the tracking tab is presented. The method utilizes a special tab material that can be remotely activated by the pilot from the cabin while in forward flight. This material is a composite consisting of shape memory alloy (SMA) wires embedded into a polymeric matrix. The activation of the SMA composite tab is done through electric current controlled from the pilot cabin and sent through conductors along the blade to the tracking tab. The proposed method will achieve in-flight tracking tab adjustment, will minimize the adjustment cycles and will reduce by at least a 
factor of ten the total tracking time. The implementation of the proposed incrementally adjustable in-flight tracking tab will rapidly minimize the once-per-rev helicopter vibration forward flight. This will lead to reduced crew fatigue, increased component life, and reduced maintenance for the helicopter. Additionally, the proposed method will also be serve as emergency system to reduce unbalanced vibrations due to ballistic damage of the rotor blade.

\section{ACKNOWLEDGMENTS}

The authors gratefully acknowledge the support of Army Research Office - University Research Initiative Program, Grant No. DAAL 03-92-0181, Dr. Gary Anderson, Program Manager.

\section{REFERENCES}

1. Bramwell, A. R. S., Helicopter Dynamics, Arnold, London, 1976

2. Straub, F. K., 1995, "Application of Smart Structures Materials Applications for Rotor Control", 2nd Army Workshop on Smart Structures and Materials, University of Maryland, College Park, MD, September 5-7, 1995.

3. Dadone, L., 1995, "An Overview of Smart Structures Materials Applications to Rotor Blades", 2nd Army Workshop on Smart Structures and Materials, University of Maryland, College Park, MD, September 5-7, 1995.

4. Giurgiutiu, V., Craig A. Rogers, C. A., and Zuidervaat, J., 1997, "Design and Preliminary Tests of an SMA Active Composite Tab", Proceedings of the SPIE's 4th Annual Symposium on Smart Structures and Materials, paper 3041-17, Catamaran Resort Hotel, San Diego, CA, 2-6 March 1997.

5. Rogers, C. A., 1990, "Active Vibration and Structural Acoustic Control of Shape Memory Alloy Hybrid Composites: Experimental Results," Journal of the Acoustical Society of America, Vol. 88, No. 6, pp. 28032811.

6. Rogers, C. A., C. R. Fuller and C. Liang, 1990, "Active Control of Sound Radiation from Panels Using Embedded Shape Memory Alloy Fibers," Journal of Sound and Vibration, Vol. 136, No. 1, pp. 164-170.

7. Rogers, C. A., C. Liang and J. Jia, 1991, "Structural Modification of Simply-Supported Laminated Plates Using Embedded Shape Memory Alloy Fibers," Journal of Computers and Structures, Vol. 38, No. 5/6, pp. 569580

8. Paine, J. S. N., C. A. Rogers, and R. A. Smith, 1995, "Adaptive Composite Materials with Shape Memory Alloy Actuators for Cylinders and Pressure Vessels," Journal of Intelligent Material Systems and Structures, Vol. 6, No. 2, pp. 210-219.

9. Chaudhry, Z. and C. A. Rogers, 1991, "Bending and Shape Control of Beams Using SMA Actuators," Journal of Intelligent Material Systems and Structures, Vol. 2, No. 4, pp. 581-602.

10. Liang, C., Davidson, F., Schetky, L. M., Straub, F. K., 1996, "Applications of Torsional Shape Memory Alloy Actuators for Active Rotor Blade Control - Opportunities and Limitations", Proceeding of the SPIE's Symposium on Smart Structures and Materials - Smart Structures and Integrated Systems Conference, San Diego, CA, 26-29 February 1996, SPIE Vol. 2717, pp. 91-100.

11. Liang, C. and C. A. Rogers, 1990, "One-Dimensional Thermo-mechanical Constitutive Relations of Shape Memory Materials," Journal of Intelligent Material Systems and Structures, Vol. 1, No. 2, pp. 207-234.

12. Jia, J. and C. A. Rogers, 1992. "Formulation of a Mechanical Model for Composites with Embedded SMA Actuators," Journal of Mechanical Design, Vol. 114, December, pp. 670-676.

13. Lagoudas, D. C., 1995, "Micro-mechanics of Shape Memory Alloys Active Composites and Structures", 2nd Workshop on Smart Structures and Materials, University of Maryland, September 5-7, 1995.

14. Wu, K., Yang, F., Pu, Z., Shi, J., 1995, "The effect of Strain Rate on the Detwinning and Superelastic Behavior of NiTi Shape Memory Alloys", Proceedings of the 6th International Congress on Adaptive Structures Technology, Key West, FL, November 1995.

15. Zhang, X. D., C. A. Rogers and C. Liang, 1992. "Modeling of the Two-Way Shape Memory Effect," Philosophical Magazine, Vol. 65, No. 5, pp. 11991215.

16. Barrett, D. J., 1995, "A One-Dimensional Constitutive Model for Shape Memory Alloys", Journal of Intelligent Material Systems and Structures, Vol. 6, May 1995, pp. 329-337.

17. Giurgiutiu, V., 1982, Elements of Helicopter Aeroelasticity - Blade Studies (in Romanian), Technical Press, Bucharest, Romania, 282 pages, 1982

18. Jia, J. and C. A. Rogers, 1992. "Formulation of a Mechanical Model for Composites with Embedded SMA Actuators," Journal of Mechanical Design, Vol. 114, December, pp. 670-676.

19. Paine, J. S. N. and C. A. Rogers, 1991, "The Effect of Thermoplastic Composite Processing on the Performance of Embedded Nitinol Actuators," Journal of Thermoplastic Composite Materials, Vol. 4, April, pp. 102-122.

20. Giurgiutiu, V., Chaudhry, Z. and Rogers, C.A., 1996a, "Energy-Based Comparison of Solid-State InducedStrain Actuators ", Journal of Intelligent Material Systems and Structures, Vol. 7, No. 1, January 1996, Technomic Pub. Co., pp. 4-14.

21. Straub, F. K., 1996, Private Communication, McDonnell Douglas Helicopter Company, Mesa, AZ.

22. Giurgiutiu, V., Chaudhry, Z., Rogers, C.A., 1994b, "Efficient Use of Induced Strain Actuators in Aeroelastic Active Control", Proceedings of the Second European Conference on Smart Structures and Materials, October 12-14, 1994, Glasgow, UK, SPIE Volume 2361, pp. 273-276.

23. Giurgiutiu, V., Chaudhry, Z., Rogers, C.A., 1995d, " Design of Displacement Amplified Induced-Strain Actuators for Maximum Energy Output ", submitted to ASME Journal of Mechanical Design 